\title{
Outcome and EBRA migration analysis of a reconstruction cage in acetabular revision arthroplasty: a clinical and radiological study
}

\author{
Dietmar Dammerer $^{1} \cdot$ Philipp Blum ${ }^{1}\left[\right.$ David Putzer² ${ }^{2}$ Annelies van Beeck ${ }^{3} \cdot$ Michael Nogler $^{2} \cdot$ Martin Thaler $^{1}$
}

Received: 18 September 2020 / Accepted: 6 December 2020 / Published online: 22 December 2020

(c) The Author(s) 2020

\begin{abstract}
Purpose Restoration cages and bone allografts have been proposed to manage severe acetabular bone defects. We aimed to investigate the migration behaviour of a restoration cup and impacted allograft bone in severe acetabular defects with Einzel-Bild-Röntgen-Analyse (EBRA).

Methods Applying a retrospective study design, 64 cases treated between 2009 and 2016 were reviewed. We determined the preoperative Charlson Comorbidity Index (CCI), pre- to postoperative WOMAC score, blood loss and functional outcome. From preoperative $\mathrm{x}$ rays, the acetabular deficiencies were classified according to Paprosky. Cup migration analyses were performed with EBRA.

Results Mean age at surgery was 73 (range: 38-93) years. According to the classification by Paprosky et al., 50\% $(n=32)$ of our patients showed a type III B and $28.1 \%(n=18)$ a type III A defect. Radiological follow-up for migration analysis was 35 (range: 4-95) months. Migration analysis showed a mean cup migration of $0.7 \mathrm{~mm}$ (range: 5.7-9.6) medial and $1.8 \mathrm{~mm}$ (range: 1.7-12.6) cranial.

Conclusion In conclusion, acetabular restoration cages in combination with bone impaction grafting showed a low revision rate at a mean follow-up of 35 months. Mean cup migration revealed low rates after 2 years and suggested a stable postoperative implant position.
\end{abstract}

Keywords Cup migration · Total hip arthroplasty · Cup revision · Restoration cup · Einzel-Bild-Röntgen-Analyse (EBRA)

Philipp Blum

philippblum1@gmx.de

Dietmar Dammerer

dietmar.dammerer@tirol-kliniken.at

David Putzer

david.putzer@i-med.ac.at

Annelies van Beeck

annelies.vanbeeck@gmail.com

Michael Nogler

michael.nogler@i-med.ac.at

Martin Thaler

martin.thaler@i-med.ac.at

1 Department of Orthopaedics and Traumatology, Medical University of Innsbruck, Anichstrasse 35, 6020 Innsbruck, Austria

2 Department of Experimental Orthopedics, Medical University of Innsbruck, Innrain 36, 6020 Innsbruck, Austria

3 Antwerp University Hospital, Wilrijkstraat 10, 2650 Edegem, Belgium

\section{Introduction}

As the number of patients receiving total hip arthroplasty (THA) grows, the number of cases requiring revision surgery increases accordingly [1]. The rate of acetabular cup revision is gradually rising and there is a heightened risk for osteolysis, prosthesis loosening over time and an increase in life expectancy [2].

Patients with failed acetabular cup revision surgery frequently have severe bone cavities and segmented defects resulting in pelvic deficiency, and present a difficult situation for revision arthroplasty [3]. Specifically, to restore the pelvic bone stock, the acetabular component should be placed in the correct anatomical position and the joint stability optimized [4]. The reconstruction should permit stable fixation of the new acetabular component and should lead to the restoration of the center of rotation $[5,6]$.

The current literature shows that substantial bone loss has been a major concern in revision THA [3, 7]. Bone impaction grafting has proven to be a helpful method for restoring 
the bone stock [8]. In cases of severe acetabular defects the use of a so-called restoration cage allows the bony acetabular defect to be bridged and protected until a bone stock has been formed [9]. The literature reports the use of restoration cages to be a valuable option in treating severe bone defects $[3,5,10]$. Although, migration analysis is a well-established method for predicting life expectancy and long-term outcome of implants in primary THA [11, 12], data regarding migration analysis in acetabular reconstruction cages are still missing. Accurate measurement of early migration of revision acetabular components is crucial to establish whether surgical techniques and implant designs are evaluated for their long-term outcome [13].

For acetabular reconstructions, reinforcement rings or restoration cages are frequently used. However, the clinical and radiological results of restoration cages are controversial $[3,5,10]$. A small number of previously published studies have reported implant success rates ranging from 58\% to $100 \%[3,5,10]$. None of those studies investigated the migration behavior of restoration cages.

The purpose of our study was to investigate the clinical outcome and perform a migration analysis of a frequently used acetabular restoration cage in revision arthroplasty of the acetabulum.

\section{Materials and methods}

The study protocol was approved by the Ethics committee of the Medical University of Innsbruck, Austria, Europe. Written informed consent was obtained from all subjects before participation. All methods and measurements were carried out in accordance with relevant guidelines and regulations.

Applying a retrospective study design with a prospective follow-up, we reviewed medical histories of all consecutive patients who received a Graft Augmentation Prosthesis (GAP) II by Stryker Orthopaedics (Mahwah, NJ, USA) at our Department between September 2009 and August 2016. The GAP II is an acetabular restoration cage and consists of a sand-blasted titanium shell with a superior-posterior lip and an inferior hook, which is used for fixation at the Köhler teardrop. Two superior plates allow screw fixation to the ilium, and screw fixation of the titanium shell to the pelvic bone is possible $[3,5]$.

During the above-mentioned period, 64 GAP II cages were implanted in 60 patients (female $=37$; male $=23$ ). In three patients the GAP II was implanted bilaterally, while one patient was revised on the same side with a GAP II after the initial GAP II prosthesis failed. Sociodemographic and surgical data are shown in Table 1.

Indications for revision surgery were aseptic cup loosening $(n=32)$, infection $(n=10)$, periprosthetic acetabulum fracture $(n=7)$, polyethylene wear $(n=5)$, protrusio of the implanted acetabular component $(n=3)$, cup malposition $(n=1)$, material and periprosthetic fracture $(n=1)$, severe hip dysplasia and secondary osteoarthritis $(n=1)$, osteoarthritis with acetabular defect $(n=1)$, periprosthetic fracture of the femur $(n=1)$ and femoral neck fracture with acetabular defect $(n=1)$. The GAP II was used once as a primary implant in a femoral head necrosis, resulting in an acetabular bone defect $(n=1)$.

From preoperative X-rays the acetabular deficiencies were classified according to Paprosky et al. [14]. Accordingly, 3.1\% $(n=2)$ showed type I, $1.6 \%(n=1)$ type II A, $12.5 \%(n=8)$ type II $\mathrm{B}, 4.7 \%$ type II C $(n=3), 28.1 \%$ type III A $(n=18)$ and $50 \%$ type III B $(n=32)$ (Table 1$)$.

Two senior consultant surgeons performed over $90 \%$ of all the surgeries mentioned in this study. Three different approaches were used: in 58 cases a direct anterior approach $[15,16]$, in five cases a lateral transgluteal approach [17] and once a dorsal approach $[18,19]$. The surgical approach was chosen in way of a) initial approach, b) the bone defect, which has to be addressed in revision surgery and c) the reachability through the chosen approach. The majority of the procedures were performed in the supine position $(n=63)$. In one case the surgery was performed in lateral decubitus.

The range of motion was pre- and postoperatively assessed with reference to the medical histories. In addition, patients were asked to complete the Western Ontario and McMaster Universities Osteoarthritis Index (WOMAC) questionnaire pre- and postoperatively [20].

The patients were followed with radiographs before discharge, six weeks after surgery and 12 months postoperatively. Additional radiographs were performed if the patient had any complaints after revision THA. All radiographs were taken in standardized conditions with the exact same technique (anterior-posterior (AP) radiographs; patient standing in the upright position and full weight-bearing) following the EBRA protocol [21, 22]. The postoperative radiographs were used to evaluate the ingrowth of the bone graft and assess the position of the hook, which is mentioned for fixation around the Köhler teardrop. Based on the radiological criteria for implant failure described by Sembrano et al. [23], a stable cup was defined as the presence of intact hooks, screws and plates, the absence of increased radiolucency in the area of the implant and an implant migration of less than $5 \mathrm{~mm}$ at the time of the last follow-up. According to the criteria of Sloof et al. [24], an additional radiological analysis of the bone graft was performed to evaluate the consolidation of the bone graft. This was defined as the presence of trabecular bone crossing the graft-host junction.

Before migration analysis, we validated EBRA in revision reconstruction cases. We took more than $30 \times$ rays following the EBRA protocol and in different pelvic tilt positions. EBRA migration analysis was performed with a 
Table 1 Sociodemographic and surgical data of the study group. The range is given in brackets

\begin{tabular}{|c|c|c|}
\hline \multirow[t]{3}{*}{ Number of patients } & Female & 37 \\
\hline & Male & 23 \\
\hline & Total & 60 \\
\hline \multirow[t]{3}{*}{ Side } & Left & 33 \\
\hline & Right & 31 \\
\hline & Total & 64 \\
\hline Mean age at surgery (years) & & $73(38-93)$ \\
\hline Body Mass Index $\left(\mathrm{kg} / \mathrm{m}^{2}\right)$ & & $26.3(18.4-44.4)$ \\
\hline \multirow[t]{2}{*}{ WOMAC Score (total) } & Preoperative & $58.5(26.1-82.9)$ \\
\hline & Postoperative & $30.8(11.2-79.2)$ \\
\hline \multirow[t]{12}{*}{ Surgical indication } & Aseptic cup loosening & 32 \\
\hline & Infection & 10 \\
\hline & Periprosthetic acetabulum fracture & 7 \\
\hline & Polyethylene wear & 5 \\
\hline & $\begin{array}{l}\text { Acetabular protrusion of former cup and aseptic } \\
\text { loosening }\end{array}$ & 3 \\
\hline & Cup malposition & 1 \\
\hline & Material and periprosthetic fracture & 1 \\
\hline & Hip dysplasia and secondary osteoarthritis & 1 \\
\hline & Osteoarthritis and severe acetabular defect & 1 \\
\hline & Periprosthetic femur fracture & 1 \\
\hline & Femoral neck fracture and acetabular defect & 1 \\
\hline & Femoral head necrosis and acetabular defect & 1 \\
\hline Operation time [min] (range) & & $163(81-332)$ \\
\hline \multirow[t]{3}{*}{ Surgical approach } & Direct anterior & 58 \\
\hline & Lateral transgluteal & 5 \\
\hline & Dorsal & 1 \\
\hline \multirow[t]{2}{*}{ Surgical position } & Supine & 63 \\
\hline & Lateral & 1 \\
\hline Calculated Blood loss [ml] & & $2348(346-4816)$ \\
\hline \multirow{6}{*}{$\begin{array}{l}\text { Classification acetabular defect } \\
\text { Paprosky }\end{array}$} & Type I & 2 \\
\hline & Type II A & 1 \\
\hline & Type II B & 8 \\
\hline & Type II C & 3 \\
\hline & Type III A & 18 \\
\hline & Type III B & 32 \\
\hline
\end{tabular}

comparability limit of $3.0 \mathrm{~mm}$ (95\% confidence interval). Negative horizontal migration values were defined as medial migration. Negative vertical migration (distal migration) up to $1 \mathrm{~mm}$ was due to the limited accuracy of the EBRA measurement method. Based on the pre-test results and together with the EBRA engineers, the measurement results were able to be validated and verified. Figure 1 shows the anatomical phantom and a patient case including EBRA reference.

Einzel-Bild-Röntgen-Analyse (EBRA) was used to measure migration of the GAP II restoration cage. Included were all patients with at least four postoperative plain hip x rays. EBRA is a well-established method that evaluates standard anterior-posterior radiographs without requiring additional means at exposure (e.g., ball markers). Simulating the spatial situation, it computes parameters of longitudinal and transverse migration of the prosthetic cup and femoral head. A comparability algorithm using a grid of transverse and longitudinal tangents of the pelvis contour divides serial radiographs into sets of comparable ones. Migration is measured only between comparable radiographs. The $95 \%$ confidence limits for EBRA results are $1.0 \mathrm{~mm}$ for longitudinal and $0.8 \mathrm{~mm}$ for transverse migration [21, 25]. EBRA migration analysis was performed $[21,25]$ by an independent investigator, who was not involved in the surgeries or postoperative treatment of the patients. 


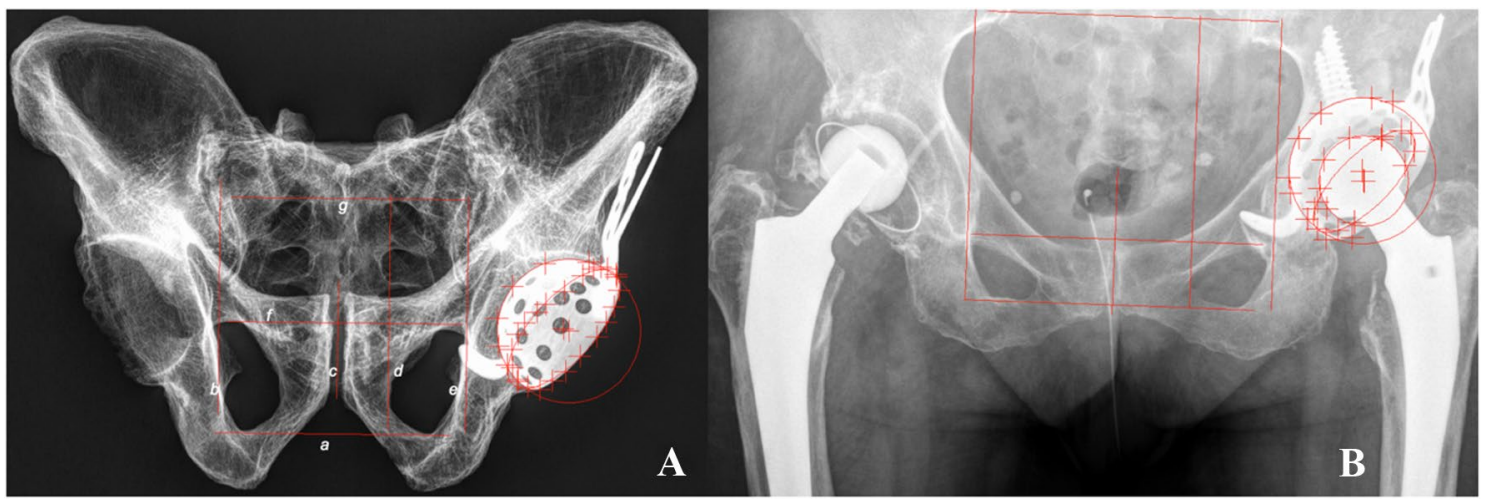

Fig. 1 Anterior-posterior $\mathrm{x}$ rays of a pelvic bone as follows: a anatomical phantom and $\mathbf{b}$ patient showing a GAP II cage and drawn tangents for migration analysis with EBRA, (a) base line, (b) right line, (c) symphysis line, (d) foramen line, (e) left line and (g) top line

\section{Statistical analysis}

An independent statistician performed the statistical analyses. For statistical analysis, Excel (Microsoft Office Professional Plus 10, Redmond, WA, USA) and Graph Pad Prism (Version 7.0, GraphPad Software Inc., La Jolla, CA, USA) were used. A descriptive statistic was calculated for all parameters, including mean, median, standard deviation, interquartile range, minimum, maximum and range. An analysis of variance, performed for repeated measurements, tested the results of the migration analysis for significant differences $(p<0.05)$. In addition, a Kaplan-Meier survival analysis was performed for the implant.

\section{Results}

The mean age at surgery was 73 (range: 38-93) years. Mean Body Mass Index was 26.3 (range: 18.4-44.4) kg/m2. Mean clinical follow-up was 29 (range: 1-96) months. Mean radiological follow-up for the EBRA migration analysis was 35 (range: 4-95) months. Average surgery time was 163 (range: 81-332) min (Table 1). According to the bone impaction grafting technique, allogenic cancellous bony chips were used in 62 of the 64 hips. The bone grafts were taken from the Department's own bone bank. Consolidation of the bone graft was observed in 59 of the 62 cases at the final radiological follow-up (Fig. 2).

In pre- and postoperative comparison we documented an improvement in the mean WOMAC score from 58.5 (range: 26.1-82.9) to 30.8 (range: 11.2-79.2). Estimated blood loss was calculated using the formula by Mercuriali [26]. Each substitution of concentrated red blood cells, administered intra- or postoperatively until the fifth day, was included in the calculation with a quantity of $280 \mathrm{ml}$ and a haematocrit of 0.54 . Calculated blood loss averaged $2348 \mathrm{ml}$ ( $\pm 1037 \mathrm{ml}$; range: $346-4816 \mathrm{ml}$ ). Three patients died 11, 22 and 42 months after implantation of the GAP II cage. None of the causes of death were related to the surgery. Further details are shown in Table 2.

The inclusion criteria for the EBRA migration analysis were fulfilled in 45 of the 64 cases. A median of five $\mathrm{x}$-rays per patient (range: 4-14) was assessed. Migration analysis showed a mean cup migration (medial and cranial) of $0.7 \mathrm{~mm}$ (range: $5.7 \mathrm{~mm}$ medial $-9.6 \mathrm{~mm}$ lateral) and $1.8 \mathrm{~mm}$ (range: $1.7 \mathrm{~mm}$ caudal-12.6 mm cranial) in 24 months after implantation. Within 24 months only two of the 45 implants showed cup migration $>5 \mathrm{~mm}$. The most severe cup migration was found in a patient presenting with an acetabular defect grade III A according to Paprosky's classification. During the entire observation period, a migration $>5 \mathrm{~mm}$ was observed in seven hips in our study group. These were distributed over the following periods: two hips between 0 and 1 year, two hips between 2 and 3 years, one hip between 4 and 5 years, one hip between 6 and 7 years and one hip between 7 and 8 years (Fig. 3).

During the above-mentioned follow-up period, only four of the 64 GAP II cages were revised. The revision was performed after 4, 6 and 31 months. These revisions were due to implant failure (broken hook; $n=2$ ), infection $(n=1)$ or aseptic loosening $(n=1)$. In one further case, revision surgery would have been necessary because of aseptic loosening after 51 months, but the patient's state of health did not allow a revision procedure. Overall, the Kaplan-Meier analysis showed an implant survival rate of $94 \%$ (CI 95\%, 82-98) after 24 months.

In three cases, the liner had to be revised after two weeks, 12 and 52 months, whereby the acetabular restoration cage was left in place. Two patients showed recurrent luxations after revision surgery. A postoperative infection was treated with DAIR (debridement, antibiotics and implant retention), thus necessitating the third-liner revision.

During the investigated follow-up period, seven of the 64 GAP II restoration cages showed a hardware failure 
Fig. 2 Preoperative radiograph of a Paprosky type III B defect (a). 35 months after surgery, stable position of the GAP II restoration cage and good consolidation of the bone graft can be observed (b)
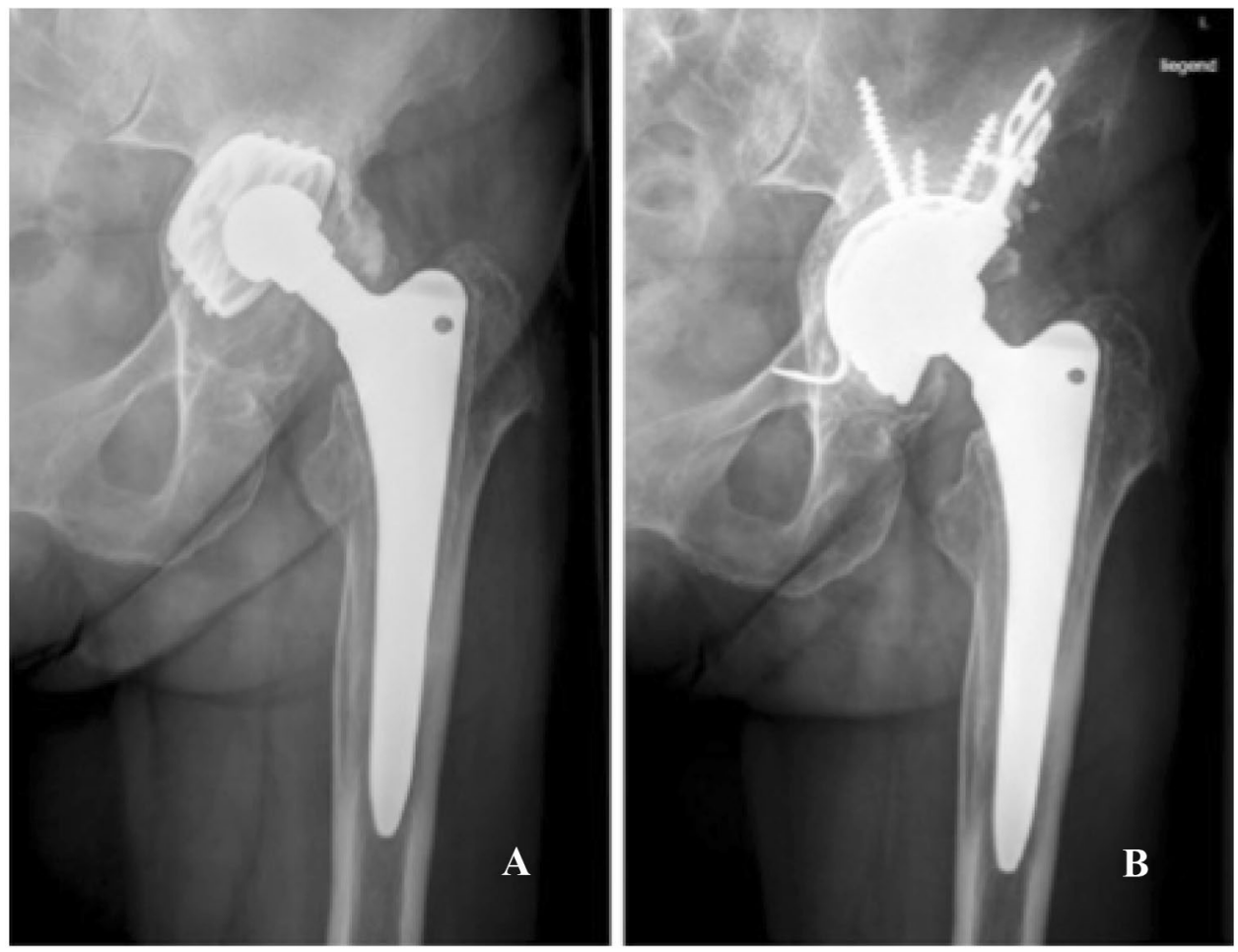

Table 2 Cohort demographics for all used cages, allogenic bone, screws, cups and results of the pre- and postoperative compares of the WOMAC score
Allogenic bone ( $n)$

Allogenic bone consolidation $(n)$

Screws used (mean; $n$ )

Mesh used (n)

Diameter and size restoration cage $(n)$
Cemented cup in cage $(n)$

WOMAC score (mean, total)

Leg length discrepancy (mean; $\mathrm{cm}$ )
$62(97 \%)$

$59(92 \%)$

5 (range; 3-7)

$13(20.3 \%)$

$5(7.8 \%)$

$\begin{array}{ll}72 \mathrm{~mm} & 5(7.8 \%) \\ 68 \mathrm{~mm} & 3(4.7 \%)\end{array}$

$64 \mathrm{~mm} \quad 11(17.2 \%)$

$60 \mathrm{~mm} \quad 18(28.1 \%)$

$56 \mathrm{~mm} \quad 21(32.8 \%)$

$54 \mathrm{~mm} \quad 1(1.5 \%)$

$52 \mathrm{~mm} \quad 4(6.2 \%)$

$50 \mathrm{~mm} \quad 1(1.5 \%)$

Müller II ${ }^{\circledR} \quad 43(67.2 \%)$

Avantage $^{\circledR} \quad 21(32.8 \%)$

Preoperative $\quad 58.5$ (range; 26.1-82.9)

Postoperative $\quad 30.8$ (range; 11.2-79.2) 1 (range; -2-3) that did not require revision surgery. A broken hook occurred in five cups. At least one broken screw was found in the five cups; none of theses cups were revised. The broken screw fixed the lateral flange of the cup to the ileum in a horizontal direction.

\section{Discussion}

Revision THA combined with a severe acetabular defect is a complex and challenging procedure [27]. A wide range 
Fig. 3 EBRA migration analysis results (medial/lateral and cranial/caudal) for the reconstruction cage
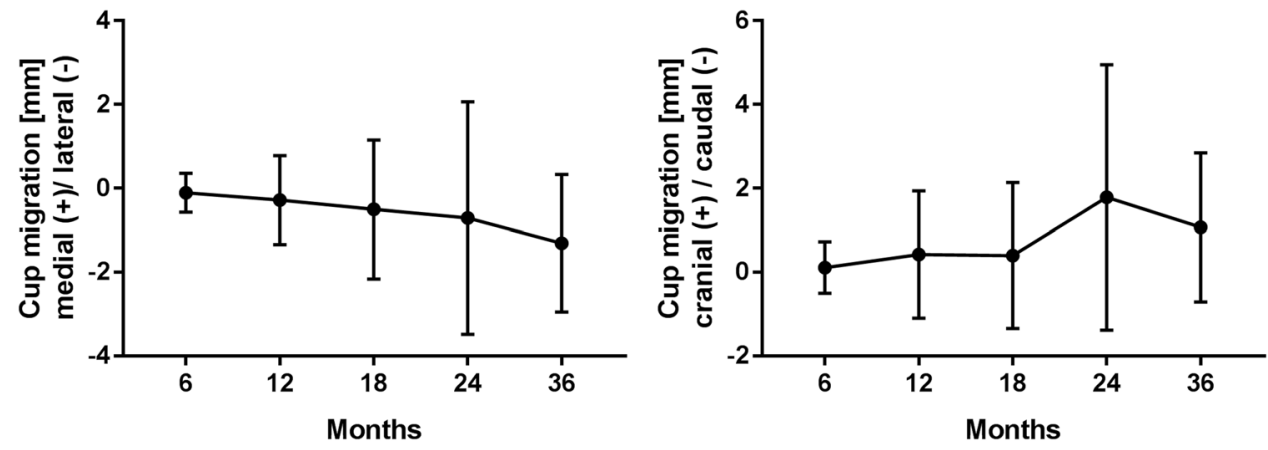

of treatment options is currently available, including the use of so-called restoration cages [28]. The purpose of the study was to perform a migration analysis of the GAP II reconstruction cage. In most cases, the GAP II cage is used with bone impaction grafting for management of acetabular defect during revision THA [3, 5, 10]. This is the first report on migration analysis of a reconstruction cage in revision THA. We also validated EBRA for migration analysis of reconstruction cages in revision THA. To the best of our knowledge, there are no published studies to date that evaluate EBRA or RSA (roentgen stereophotogrammetric analysis) in a revision setting [3, 5, 10].

Clinically published data show controversial success rates ranging from 60 to $100 \%$ after implantation of the GAP II cup [3, 5, 10] Inconsistency of implant survival can be related to the implant itself, as well as to the surgeon, the surgical technique, the chosen outcome assessments, statistical analysis, or the quality of the published study designs $[3,5,10]$. Therefore, objective parameters like migration analysis (e.g. EBRA) or RSA should be applied.

In our study, an average of $0.7 \mathrm{~mm}$ medial and $1.8 \mathrm{~mm}$ cranial cup migration was observed two years after surgery. In their publication Buttaro et al. prescribed an average medial and cranial migration of $1.4 \mathrm{~mm}$ and $2.2 \mathrm{~mm} \mathrm{[3].}$ Hosny et al. measured a mean migration of $1.9 \mathrm{~mm}$ medial and $2.1 \mathrm{~mm}$ cranial in their study according to the method by Nunn et al. [5, 29]. Both studies show greater cup migration than we found in our results. Several methods have been described to determine the migration of the acetabular component [21, 29-31]. We used the more accurate and well established EBRA method [21]. EBRA provides results with an accuracy of $\pm 1 \mathrm{~mm}[21]$.

Phillips et al. [32] confirmed the high measurement accuracy of EBRA by comparison to that of other methods. One method for migration measurement of acetabular components is the RSA [33]. The precondition for this is the perioperative implantation of tantalum balls in the pelvic bone near the acetabular cup. Two radiographs taken from different angles are needed for migration analysis. These $\mathrm{x}$ rays of the pelvis are taken in a special calibration cage in order to enable 3-dimensional evaluation with the appropriate software for measuring implant migration. As tantalum balls must be implanted, this type of migration analysis involves a major effort and cannot be performed retrospectively [30, 32, 33]. Abraham showed that EBRA can accurately measure migration of uncemented acetabular components used at revision THA [34] and that EBRA cup and RSA measurements had a good agreement on the classification of components above and below $1 \mathrm{~mm}$ at 2 years with a sensitivity and specificity of $100 \%$ and $87 \%$, respectively [34]. In cases of pelvic discontinuity and the use of augments and cages, the accuracy of EBRA migration measurements was significantly poorer [34]. However, Abraham published only three cases, where a cage was used as a "cup-cage construct" with or without an augment [34]. Our cohort consisted only of reconstruction cages used with bone graft and the number of investigated cases is 64 , representing the largest series in the literature to date.

Duffy et al. [10] presented a first small study including 17 hips and a 58\% success rate for the implant at a mean follow-up of 60 months. In their study, five implants had to be revised within 60 months, with four of the five revisions based on bone graft resorption [10]. We believe graft resorption was a cause of stress shielding and no bearing led to micromotion or played a major role in these failures [3]. The absence of structural bone may also explain these fatigue failures [3]. Nevertheless, Duffy et al. [10] recommended in their study that this implant be used only in the presence of a sufficiently stable bone stock situation. Buttaro et al. [3] abandoned further use of the GAP II restoration cage. In their study, nine of their 24 GAP II cages failed at a mean follow-up of 34 months, with six of the nine failed implants showing septic loosening [3]. In the study by Buttaro et al. the bone stock was replenished using bone impaction grafting according to the method described by Sloof et al. [3, 24]. Contrary to these results, Hosny et al. [5] observed a success rate of $100 \%$ at a mean follow-up of 49 months. A stable implant position is a precondition for the incorporation of the bone graft and bony ingrowth of the restoration cage [35, 36]. Otherwise, early migration of the implant leads to lysis of the bone graft, aseptic loosening and thus failure of the implant may occur [37]. 
This study has several limitations. First, it was a retrospective study without a control group. As a result, no statistical comparison of outcomes or cup migration with a control group using other implants was possible. Second, our study cohort was small. Still, these were not common cases, and even in high-volume centers the numbers were relatively small. Nevertheless, as far as we know, this is currently the largest study cohort in the literature using the GAP II in revision surgery. Third, we did not perform radiostereometric studies, which would likely detect earlier migration and perhaps predict some failures among those patients, who were functioning well at the time of the latest follow-up.

In summary, reinforcement rings and bone allografts have been proposed for the management of severe acetabular bone defects in revision hip surgery. The GAP II reconstruction cage showed in combination with bone impaction grafting low migration and revision rates at a mean follow-up of 35 months. In our study, the GAP II cage seems to be a good therapeutic option for revisions in THA with a severe acetabular defect. In addition, our results of the validation in an anatomical phantom model of the reconstruction cage revealed that EBRA cup analysis can now also be applied for reconstruction cages in revision THA. This will significantly increase the use of EBRA in revision arthroplasty and be an objective tool in the outcome and migration analysis.

Continued observation is necessary in order to observe the long-term success rates and follow radiologically conspicuous implants. Overall, precise preoperative evaluation of the patient as well as a patient-specific decision on the surgical procedure and the used implant is always required to ensure good mechanical stability of the implant and functionality of THA.

Authors' contributions DD and PB wrote the main manuscript. DP made the statistical analysis. AB prepared figures and tables. MN and MT reviewed and proof-read the manuscript. All authors have reviewed and confirmed the accuracy of the whole manuscript.

Funding Open Access funding provided by University of Innsbruck and Medical University of Innsbruck. This research did not receive any specific grant from funding agencies in the public, commercial, or not-for-profit sectors.

Availability of data and material Data will be sent if necessary.

\section{Compliance with ethical standards}

Conflict of interest The authors Martin Thaler and Michael Nogler have received research grants from Stryker that are not related to the content of the study. Martin Thaler is Treasurer of the European Hip Society. Dietmar Dammerer, Philipp Blum, David Putzer and Annelies van Beeck declare that they have no conflict of interest.

Code availability Not applicable.
Consent to participate Informed consent was obtained from all participants.

Consent for publication All authors have reviewed and confirmed the accuracy of the whole manuscript.

Ethics approval The local ethics committee approved the study.

Open Access This article is licensed under a Creative Commons Attribution 4.0 International License, which permits use, sharing, adaptation, distribution and reproduction in any medium or format, as long as you give appropriate credit to the original author(s) and the source, provide a link to the Creative Commons licence, and indicate if changes were made. The images or other third party material in this article are included in the article's Creative Commons licence, unless indicated otherwise in a credit line to the material. If material is not included in the article's Creative Commons licence and your intended use is not permitted by statutory regulation or exceeds the permitted use, you will need to obtain permission directly from the copyright holder. To view a copy of this licence, visit http://creativecommons.org/licenses/by/4.0/.

\section{References}

1. Makela KT, Eskelinen A, Pulkkinen P, Paavolainen P, Remes V (2008) Total hip arthroplasty for primary osteoarthritis in patients fifty-five years of age or older. An analysis of the Finnish arthroplasty registry. J Bone Joint Surg Am 90:2160-2170. https://doi. org/10.2106/JBJS.G.00870

2. Lee JM, Kim TH (2018) Acetabular cup revision arthroplasty using morselized impaction allograft. Hip Pelvis 30:65-77. https ://doi.org/10.5371/hp.2018.30.2.65

3. Buttaro MA, de la Rosa DM, Comba F, Piccaluga F (2012) High failure rate with the GAP II ring and impacted allograft bone in severe acetabular defects. Clin Orthop Relat Res 470:3148-3155. https://doi.org/10.1007/s11999-012-2402-8

4. Morales De Cano JJ, Guillamet L, Perez Pons A (2019) Acetabular reconstruction in paprosky type Iii defects. Acta Ortop Bras 27:59-63. https://doi.org/10.1590/1413-785220192701187313

5. Hosny HAH, El-Bakoury A, Fekry H, Keenan J (2018) Mid-term results of graft augmentation prosthesis II cage and impacted allograft bone in revision hip arthroplasty. J Arthroplasty 33:14871493. https://doi.org/10.1016/j.arth.2017.11.060

6. Sheth NP, Nelson CL, Springer BD, Fehring TK, Paprosky WG (2013) Acetabular bone loss in revision total hip arthroplasty: evaluation and management. J Am Acad Orthop Surg 21:128-139. https://doi.org/10.5435/JAAOS-21-03-128

7. Ballester Alfaro JJ, Sueiro Fernandez J (2010) Trabecular Metal buttress augment and the Trabecular Metal cup-cage construct in revision hip arthroplasty for severe acetabular bone loss and pelvic discontinuity. Hip Int 20(Suppl 7):S119-127. https://doi. org/10.5301/HIP.2010.5297

8. Ibrahim MS, Raja S, Haddad FS (2013) Acetabular impaction bone grafting in total hip replacement. Bone Joint J 95:98-102. https://doi.org/10.1302/0301-620x.95b11.32834

9. Gross AE, Goodman S (2004) The current role of structural grafts and cages in revision arthroplasty of the hip. Clin Orthop Relat Res. https://doi.org/10.1097/01.blo.0000149822.49890.5e

10. Duffy GP, O'Connor MI, Brodersen MP (2007) Fatigue failure of the GAP ring. J Arthroplasty 22:711-714. https://doi. org/10.1016/j.arth.2006.12.108

11. Nunag P, Deakin AH, Oburu E, Sarungi M (2012) Two-year radiologic assessment of the trident peripheral self-locking 
cup using EBRA. Hip Int 22:511-515. https://doi.org/10.5301/ HIP.2012.9744

12. Stihsen C, Rath C, Radl R, Saalabian AA, Materna W, Rehak P, Windhager R (2013) Early migration characteristics of a 180 degrees porous-coated cup with 1-mm press fit. Arch Orthop Trauma Surg 133:707-712. https://doi.org/10.1007/s0040 2-013-1713-X

13. Malak TT, Broomfield JA, Palmer AJ, Hopewell S, Carr A, Brown C, Prieto-Alhambra D, Glyn-Jones S (2016) Surrogate markers of long-term outcome in primary total hip arthroplasty: a systematic review. Bone Joint Res 5:206-214. https://doi.org/10.1302/20463758.56.2000568

14. Paprosky WG, Perona PG, Lawrence JM (1994) Acetabular defect classification and surgical reconstruction in revision arthroplasty. A 6-year follow-up evaluation. J Arthroplasty 9:33-44. https://doi. org/10.1016/0883-5403(94)90135-x

15. Rachbauer F (2005) Minimally invasive total hip arthroplasty via direct anterior approach. Orthopade 34:1103-1104. https://doi. org/10.1007/s00132-005-0854-1

16. Meermans G, Konan S, Das R, Volpin A, Haddad FS (2017) The direct anterior approach in total hip arthroplasty: a systematic review of the literature. Bone Joint J 99:732-740. https://doi. org/10.1302/0301-620x.99b6.38053

17. Bauer R, Kerschbaumer F, Poisel S, Oberthaler W (1979) The transgluteal approach to the hip joint. Arch Orthop Trauma Surg 95:47-49. https://doi.org/10.1007/bf00379169

18. Moretti VM, Post ZD (2017) Surgical approaches for total hip arthroplasty. Indian J Orthop 51:368-376. https://doi.org/10.4103/ ortho.IJOrtho_317_16

19. Higgins BT, Barlow DR, Heagerty NE, Lin TJ (2015) Anterior versus posterior approach for total hip arthroplasty, a systematic review and meta-analysis. J Arthroplasty 30:419-434. https://doi. org/10.1016/j.arth.2014.10.020

20. Bellamy N, Buchanan WW (1986) A preliminary evaluation of the dimensionality and clinical importance of pain and disability in osteoarthritis of the hip and knee. Clin Rheumatol 5:231-241. https://doi.org/10.1007/bf02032362

21. Krismer M, Bauer R, Tschupik J, Mayrhofer P (1995) EBRA: a method to measure migration of acetabular components. J Biomech 28:1225-1236

22. Krismer M, Stockl B, Fischer M, Bauer R, Mayrhofer P, Ogon M (1996) Early migration predicts late aseptic failure of hip sockets. J Bone Joint Surg Br 78:422-426

23. Sembrano JN, Cheng EY (2008) Acetabular cage survival and analysis of factors related to failure. Clin Orthop Relat Res 466:1657-1665. https://doi.org/10.1007/s11999-008-0183-x

24. Slooff TJ, Buma P, Schreurs BW, Schimmel JW, Huiskes R, Gardeniers J (1996) Acetabular and femoral reconstruction with impacted graft and cement. Clin Orthop Relat Res. https://doi. org/10.1097/00003086-199603000-00013

25. Krismer M, Tschupik JP, Bauer R, Mayrhofer P, Stockl B, Fischer M, Biedermann R (1997) Single-image roentgen analysis for the measurement of hip endoprosthesis migration. Orthopade 26:229-236. https://doi.org/10.1007/s001320050089

26. Meunier A, Petersson A, Good L, Berlin G (2008) Validation of a haemoglobin dilution method for estimation of blood loss. Vox Sang 95:120-124. https://doi.org/10.111 1/j.1423-0410.2008.01071.x

27. Jain S, Grogan RJ, Giannoudis PV (2014) Options for managing severe acetabular bone loss in revision hip arthroplasty. A systematic review. Hip Int 24:109-122. https://doi.org/10.5301/ hipint.5000101

28. Kim YH (2017) Acetabular cup revision. Hip Pelvis 29:155-158. https://doi.org/10.5371/hp.2017.29.3.155

29. Nunn D, Freeman MA, Hill PF, Evans SJ (1989) The measurement of migration of the acetabular component of hip prostheses. J Bone Joint Surg Br 71:629-631

30. Ilchmann T, Franzen H, Mjoberg B, Wingstrand H (1992) Measurement accuracy in acetabular cup migration. A comparison of four radiologic methods versus roentgen stereophotogrammetric analysis. J Arthroplasty 7:121-127. https://doi.org/10.1016/08835403(92)90004-a

31. Sutherland CJ, Bresina SJ (1992) Measurement of acetabular component migration using two-dimensional radiography. J Arthroplasty 7(Suppl):377-379. https://doi.org/10.1016/s0883 -5403(07)80027-1

32. Phillips NJ, Stockley I, Wilkinson JM (2002) Direct plain radiographic methods versus EBRA-Digital for measuring implant migration after total hip arthroplasty. J Arthroplasty 17:917-925. https://doi.org/10.1054/arth.2002.34529

33. Selvik G (1990) Roentgen stereophotogrammetric analysis. Acta Radiol 31:113-126

34. Abrahams JM, Callary SA, Jang SW, Hewitt J, Howie DW, Solomon LB (2020) Accuracy of EBRA-cup measurements after reconstruction of severe acetabular defects at revision THR. J Orthop Res. https://doi.org/10.1002/jor.24623

35. Makinen TJ, Fichman SG, Watts E, Kuzyk PR, Safir OA, Gross $\mathrm{AE}$ (2016) The role of cages in the management of severe acetabular bone defects at revision arthroplasty. Bone Joint J 98:73-77. https://doi.org/10.1302/0301-620x.98b1.36307

36. Paprosky WG, Sporer SS, Murphy BP (2007) Addressing severe bone deficiency: what a cage will not do. J Arthroplasty 22:111115. https://doi.org/10.1016/j.arth.2007.01.018

37. Deirmengian GK, Zmistowski B, O’Neil JT, Hozack WJ (2011) Management of acetabular bone loss in revision total hip arthroplasty. J Bone Joint Surg Am 93:1842-1852. https://doi. org/10.2106/JBJS.J.01197

Publisher's Note Springer Nature remains neutral with regard to jurisdictional claims in published maps and institutional affiliations. 